\title{
Influencia de los sistemas de iniciación en la entrega de energía de los explosivos pentolita, PTX-2 y composición B
}

\author{
Influence of initiation systems on the energy delivery of pentolite, PTX-2 and composition B explosives
}

\author{
Jorge Fonseca $^{a}$;John Rincón ${ }^{a}$; Héctor Alvarado ${ }^{a}$; Lina Murcia ${ }^{a}$ \\ ${ }^{a}$ Escuela de Ingenieros Militares, Colombia
}

Correspondencia: Jorge.fonsecab@esing.edu.co, ifrinconf@gmail.com

Recibido: Mayo 11, 2021. Aceptado: Junio 10, 2021. Publicado: Junio 12, 2021

\begin{abstract}
Resumen
Se inicia con información bibliográfica para la realización experimental de las características técnicas (presión detonación, sensibilidad y velocidad de detonación) de los explosivos militares, para determinar su influencia en la entrega de energía al emplear sistemas de iniciación o cápsulas diferentes, como son la de fabricación peruana, china y fuerza 12. El método de investigación empleado es la fabricación de los explosivos a nivel laboratorio y el análisis de los resultados de los ensayos obtenidos de las características técnicas de los explosivos en relación a los diferentes medios de iniciación anteriormente mencionados como son las cápsulas. Finalmente; se muestra los resultados obtenidos de las características técnicas para cada uno de los explosivos militares, permitiendo correlacionar estas características con el desempeño balístico en entrega de energía, dependiendo esta entrega principalmente del medio de iniciación elegido. Al final, del artículo, se muestra el medio de iniciación a emplear para los explosivos militares pentolita, PTX-2 y composición B.
\end{abstract}

Palabras claves: Explosivo; Sistema iniciación; Presión de detonación; Velocidad de detonación; sensibilidad.

\section{Introducción}

Un criterio de selección de los explosivos es el tipo de trabajo a realizar, pudiéndose clasificar estos materiales frente a su uso en ámbitos "a nivel civil (industrial) y/o militar" [1]. Actualmente, los explosivos militares se emplean en actividades de demolición por su alto contenido de energía (poder rompedor) requiriendo estos un adecuado sistema de iniciación como son las cápsulas. Entre los explosivos militares más representativos encontramos la Pentolita, PTX-2 y composición B [2].

Las actividades de demolición, además de requerir sistemas de iniciación, necesitan el estudio de materiales con características técnicas como poder rompedor (presión de detonación (PD)), velocidad de detonación ( $\mathrm{VoD})$ y sensibilidad a sistemas o cápsulas de iniciación (prueba de péndulo [3].

\begin{abstract}
It begins with bibliographic information for the experimental realization of the technical characteristics (detonation pressure, sensitivity and detonation velocity) of military explosives, to determine their influence on the delivery of energy by using initiation systems or different capsules, such as manufacturing Peruvian, Chinese and strength 12The research method used is the manufacture of explosives at the laboratory level and the analysis of the results of the tests obtained from the technical characteristics of the explosives in relation to the different initiation media mentioned above how are the capsules. Finally; the results obtained from the technical characteristics for each of the military explosives are shown, allowing these characteristics to be correlated with ballistic performance, in energy delivery depending on this energy delivery mainly from the chosen initiation medium. At the end of the article, the initiation medium to be used for military explosives pentolite, PTX-2 and composition B is shown.
\end{abstract}

Keywords: Explosive; Initiation system; Detonation pressure; Detonation speed; sensitivity.

Las características técnicas de los explosivos analizadas mediante un sistema de iniciación o cápsula algunas veces son reportadas o no por textos o catálogos de fabricantes. Un ejemplo de dichas características frente a un sistema de iniciación son las que publica la Industria Militar (Indumil) como fabricante en Colombia utilizando como cápsula el detonador común \#8 en relación al explosivo Pentolita [3]. La publicación de las características técnicas de todos los explosivos mediante un determinado sistema de iniciación no se conoce regularmente, siendo obligatorio el estudio e investigación. En este artículo, se comienza con la formulación, producción de explosivos a nivel de laboratorio, para así posteriormente evaluar las características técnicas frente a un sistema de iniciación de los siguientes explosivos: tres mezclas de composición B, PTX-2 y Pentolita, tomando este último explosivo como material de referencia. 
En consecuencia, se presenta en este artículo la realización de ensayos para el estudio de dichas características técnicas manteniendo constante otras variables, entre estas, diámetro de confinamiento, composición química y cantidad de explosivo a usar.

Los ensayos hechos son presión de detonación (prueba de Hess), velocidad de detonación y sensibilidad a la cápsula (detonador) o sistema de iniciación (prueba de péndulo), con el fin de determinar y evaluar las características técnicas y desempeño de estos materiales en cuanto la entrega de energía al cambiar su sistema de iniciación. Los sistemas de iniciación empleados en este trabajo son: 1) cápsula iniciación común \#8 de fabricación peruana y china con mecha de seguridad y 2) cápsula iniciación sísmica eléctrica fuerza 12 con mecha de seguridad.

La presión de detonación (prueba de Hess) consiste en medir la energía del explosivo liberada al ocurrir la detonación, la cual será utilizada para realizar el trabajo en la roca o material a volar e iniciar su fracturamiento. Este ensayo consiste en detonar $50 \mathrm{~g}$ de explosivo para deformar un cilindro de un determinado material y longitud [4], en donde el diámetro del explosivo será igual al diámetro del cilindro.

El ensayo velocidad de detonación ( VoD), mide la velocidad de la onda generada por el explosivo en metros por segundo a lo largo de una columna explosiva, sea al aire libre o en confinamiento en una cavidad cilíndrica conocida como barreno en un diseño de voladura. (Instituto Tecnológico Geo minero de España, 1994). Los factores que afectan, la velocidad de detonación, son la densidad de carga, diámetro, confinamiento y el envejecimiento del explosivo. La velocidad depende principalmente de su confinamiento o diámetro, siendo ello, una variable crítica en el ensayo [4].

La prueba del péndulo consiste en la medición de una elongación de este dispositivo producida por la energía del explosivo al ser este detonado mediante un sistema de iniciación [3].

Finalmente, la comparación y análisis de los resultados de los ensayos anteriores, permitirán al usuario la selección adecuada de su sistema de iniciación en un determinado explosivo con el fin de aprovechar la máxima entrega de energía y desempeño balístico de este material en una determinada demolición.

\section{Método}

Se empleó como metodología las siguientes fases: 1) la elaboración de los explosivos militares en sus respectivas composiciones químicas, 2) la realización de los ensayos ( prueba de HESS, velocidad de detonación y prueba de péndulo) y 3) el análisis de los resultados experimentales obtenidos en los ensayos para evaluación de las características técnicas de los explosivos, mediante comparación, para la estimación de entrega de energía de los explosivos al emplear un sistema de iniciación elegido.

\subsection{Elaboración y composición química de los explosivos}

La mayoría de los explosivos militares son mezclas; necesitando para su elaboración la participación de otros explosivos o sustancias químicas [5]. A continuación, en la Tab. 1, se presenta la composición química en porcentaje de los explosivos y posteriormente el procedimiento empleado a nivel de laboratorio [6], para la fabricación de estos en una composición química elegida.

Tabla 1. Composición química (\%) de los explosivos militares

\begin{tabular}{cccccc}
\hline $\begin{array}{c}\text { Sustancia } \\
\text { explosiva } \\
\text { y/o } \\
\text { química }\end{array}$ & Pentolita & PTX-2 & $\begin{array}{c}\text { Composi } \\
\text { ción B } \\
\text { mezcla-I }\end{array}$ & $\begin{array}{c}\text { Composi } \\
\text { ción B } \\
\text { mezcla-II }\end{array}$ & $\begin{array}{c}\text { Composi } \\
\text { ción B } \\
\text { mezcla- } \\
\text { III }\end{array}$ \\
\hline TNT & $50 \%$ & $44 \%$ & $60 \%$ & $39 \%$ & $49,5 \%$ \\
\hline PETN & $50 \%$ & $28 \%$ & --- & --- & --- \\
\hline RDX & --- & $28 \%$ & $39 \%$ & $60 \%$ & $49,5 \%$ \\
\hline CERA & --- & --- & $1 \%$ & $1 \%$ & $1 \%$ \\
\hline & & & & \\
\hline
\end{tabular}

\subsubsection{Procedimiento}

Para comenzar, se determina las propiedades químicas y físicas de la sustancia química o explosiva antes de elaborar el explosivo militar [7]. Entre las propiedades químicas o físicas de estas sustancias se destacan la pureza, punto de fusión y color. Asimismo se procede a estimar su cantidad en peso por medio de una balanza analítica de acuerdo a la composición química de la Tab. 1.

Posteriormente en un vaso de precipitado, se comienza a fundir lentamente el TNT a $85^{\circ} \mathrm{C}$, para así agregar e incorporar las demás sustancias químicas o explosivos hasta conformar una mezcla homogénea [8].

Por último, se dosifica la mezcla homogénea (explosivo militar) en elementos como contenedores de cartón para la realización de la prueba de Hess, cargas lineales con masa explosiva para la determinación de $\mathrm{VoD}$ y frascos de vidrio para la prueba de péndulo. A los anteriores elementos se deja un orificio para el acople del sistema de iniciación.

\subsection{Realización de los ensayos}

\subsubsection{Prueba de Hess}

La prueba de Hess se utiliza para estimar el poder rompedor de un explosivo [9], de acuerdo a la deformación producida por la detonación de una determinada cantidad de explosivo sobre un cilindro. La presión de detonación está directamente relacionada con la densidad y VoD del explosivo. Con los resultados obtenidos en esta prueba se compara el poder de un explosivo en relación a otro.

\subsubsection{Procedimiento}

En principio, se toma y se coloca un cilindro de aluminio de $25 \mathrm{~mm}$ de diámetro por $50 \mathrm{~mm}$, en una placa de hierro de $12,75 \mathrm{~mm}$ de espesor y un área de $100 \mathrm{~mm}$ de largo y 100 
$\mathrm{mm}$ de ancho. Posteriormente a la muestra confinada de explosivo, tubo de cartón, se acopla un sistema de iniciación (cebado); y a este conjunto, se ensambla al cilindro de aluminio; quedando el montaje, tal como se ilustra en la Fig. 1 .

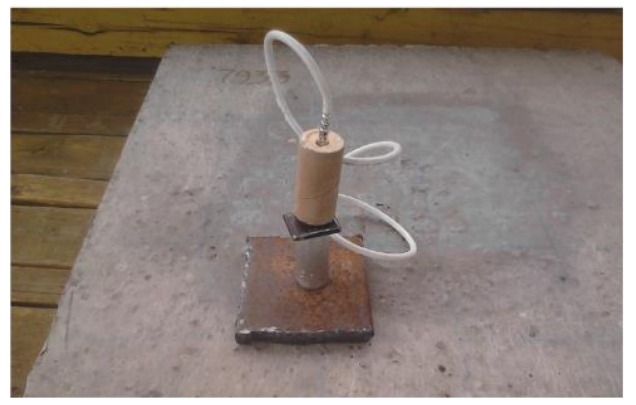

Figura 1. Prueba de Hess. Montaje y cebado

Finalmente; se inicia el explosivo, causando en el cilindro una deformación. Dicha deformación se mide para estimar el poder rompedor del material explosivo (Fig. 2 y 3).

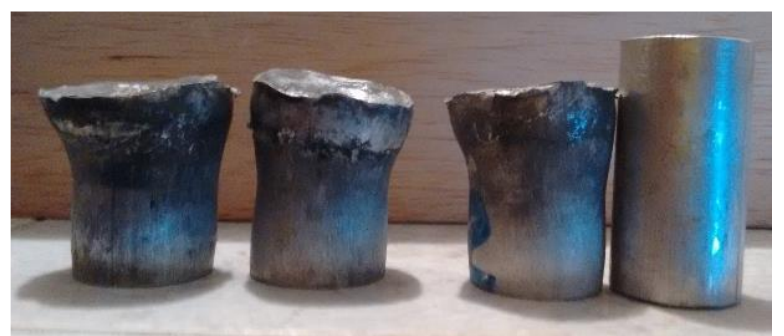

Figura 2. Prueba de Hess. Deformación producida por el explosivo.

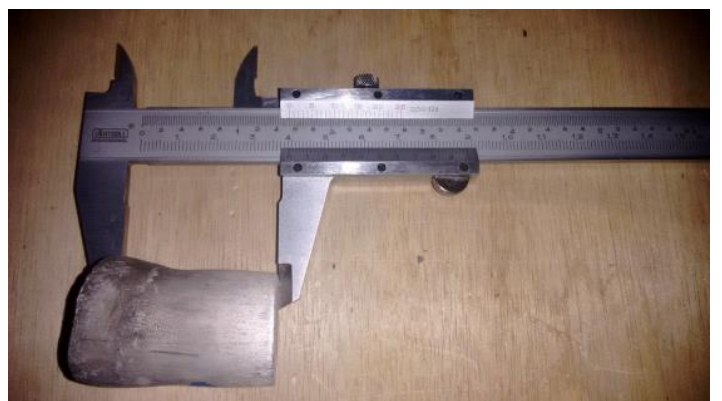

Figura 3. Prueba de Hess. Medición de la deformación.

\subsection{Prueba de velocidad de detonación}

Para la velocidad de detonación (VoD) se utiliza un contador de tiempo, el cual mide el tiempo que recorre la detonación entre dos puntos A y B.

\subsubsection{Procedimiento}

Al iniciar la carga lineal por el sistema de iniciación, la onda de detonación del explosivo, pasa por el punto A y comienza el contador a contabilizar el tiempo y al llegar al punto B se detiene. Luego este se calcula por la formula $\mathrm{V}=\mathrm{d} / \mathrm{t}$ (velocidad $=$ distancia/tiempo) $[10]$. A continuación se ilustra su medición, Fig. 4, para una carga lineal de pentolita, mediante el empleo de dos conectores eléctricos, perteneciendo estos a los puntos A y B y marcando ellos la distancia a recorrer por la onda de detonación, generada por el explosivo.

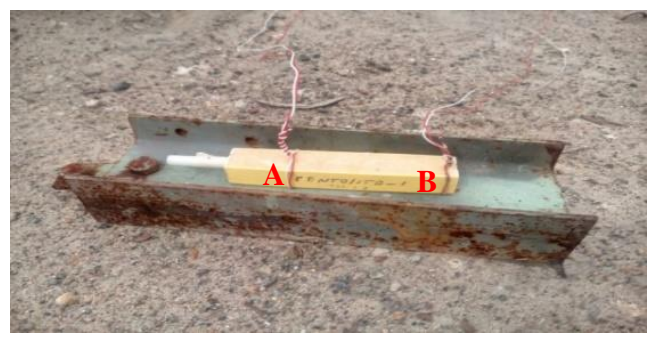

Figura 4. Medición de VoD carga lineal de Pentolita.

\subsection{Prueba de péndulo}

Consiste, en la medición de una elongación. Dicha elongación es generada por la energía del explosivo confinado [11], en un frasco de vidrio, Fig. 5, al ser este iniciado o detonado por un determinado sistema de iniciación ya sea no eléctrico, eléctrico, electrónico o con detonador común y mecha de seguridad.

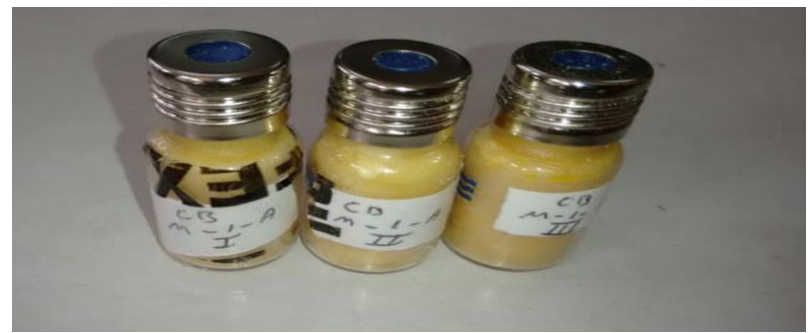

Figura 5. Explosivo Confinado

\subsubsection{Procedimiento}

El péndulo es un equipo conformado por una estructura metálica y un bloque cilíndrico con un orificio concéntrico. En dicho orificio se coloca la cantidad de masa explosiva confinada en un frasco a detonar para producir en el bloque un movimiento tipo elongación.

La prueba comienza al insertar en el agujero central del bloque cilíndrico una cantidad de explosivo confinado en un frasco de vidrio con su sistema de iniciación. Cuando el explosivo detona, genera un movimiento del bloque cilíndrico junto con el desplazamiento de un esfero (Fig. 6, parte superior izquierda) registrando el movimiento del bloque cilíndrico y el esfero sobre una cartulina, al demarcar la distancia o elongación recorrida por el conjunto bloque cilíndrico y esfero. 


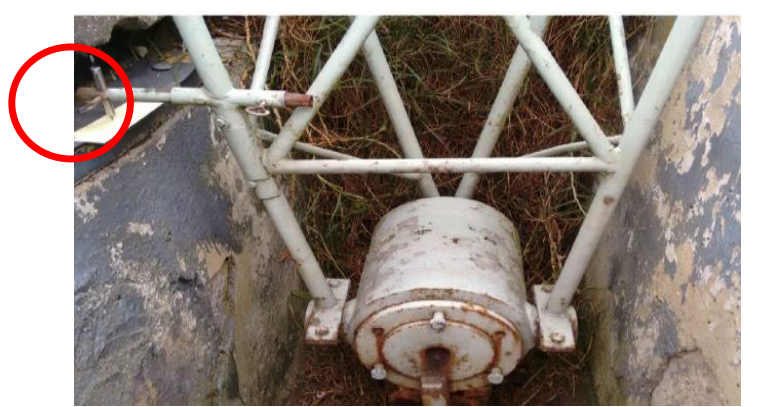

Figura 6. Equipo péndulo. Punto de marcación del desplazamiento. Fuente: Autores

\section{Resultados}

A continuación se ilustra los resultados de las características técnicas del desempeño balístico de los explosivos militares. En la tablas 2 y 3 se presenta los resultados de la prueba de HESS, tomando para estas pruebas 50 gramos de explosivo e indicando el poder rompedor del explosivo en relación a los sistemas o cápsulas de iniciación de fabricación peruana, china y fuerza 12.

El análisis de los datos Tab. 2 con el sistema de iniciación peruano, el explosivo militar que produjo mayor $\%$ deformación al cilindro fue PTX-2 con 25,90\% y el de menor deformación al cilindro fue composición B mezcla I con 5,78 $\%$. Los explosivos mezclas de composición B solo deformo el cilindro la mezcla I, las otras no deformaron el cilindro. El rango de porcentaje de deformación del cilindro para los explosivos militares fue de $5,78 \%$ y $25,90 \%$.

Tabla 2. Resultados prueba de HESS, capsula de iniciación peruana

\begin{tabular}{|c|c|c|c|}
\hline Explosivo & $\begin{array}{l}\text { Tipo de } \\
\text { cápsula de } \\
\text { iniciación }\end{array}$ & $\begin{array}{l}\text { Deformación } \\
\text { producida } \\
(\mathrm{mm})\end{array}$ & $\begin{array}{l}\% \text { Deformación } \\
\text { Producida }(*)\end{array}$ \\
\hline $\begin{array}{l}\text { Composición } \\
\text { B mezcla I }\end{array}$ & $\begin{array}{l}\text { No.8 } \\
\text { peruano }\end{array}$ & $47,36 \mathrm{~mm}$ & $5,78 \%$ \\
\hline $\begin{array}{l}\text { Composición } \\
\text { B mezcla II }\end{array}$ & $\begin{array}{l}\text { No. } \\
\text { peruano }\end{array}$ & $50,25 \mathrm{~mm}$ & $0,00 \%$ \\
\hline $\begin{array}{l}\text { Composición } \\
\text { B mezcla III }\end{array}$ & $\begin{array}{l}\text { No.8 } \\
\text { peruano }\end{array}$ & $50,51 \mathrm{~mm}$ & $0,00 \%$ \\
\hline PTX 2 & $\begin{array}{l}\text { No.8 } \\
\text { peruano }\end{array}$ & $37,43 \mathrm{~mm}$ & $25,90 \%$ \\
\hline Pentolita & $\begin{array}{l}\text { No.8 } \\
\text { peruano }\end{array}$ & $47,12 \mathrm{~mm}$ & $6,56 \%$ \\
\hline
\end{tabular}

(*) El valor de \% de deformación es producto de réplicas de tres ensayos

La Tab. 3, muestra los resultados de los explosivos militares en relación a los sistemas de iniciación chino y fuerza 12. El explosivo que presento mayor deformación al cilindro con la cápsula de iniciación No. 8 china fue pentolita con $22,15 \%$ y el de menor porcentaje de deformación fue composición B mezcla II con $7,91 \%$.
Tabla 3. Resultados prueba de HESS, capsulas de iniciación china y fuerza 12

\begin{tabular}{llll}
\hline & Tipo de & & \\
cápsula o & Deformación & $\%$ \\
Explosivo & $\begin{array}{l}\text { medio de } \\
\text { iniciación }\end{array}$ & producida $(\mathrm{mm})$ & $\begin{array}{l}\text { Deformación } \\
\text { producida }(*)\end{array}$
\end{tabular}

\begin{tabular}{|c|c|c|c|}
\hline \multirow{2}{*}{$\begin{array}{l}\text { Composición B } \\
\text { mezcla I }\end{array}$} & $\begin{array}{l}\text { No. } 8 \\
\text { Chino } \\
\end{array}$ & $41,79 \mathrm{~mm}$ & $17,89 \%$ \\
\hline & $\begin{array}{l}\text { Sísmico } \\
\text { fuerza } 12\end{array}$ & $40,53 \mathrm{~mm}$ & $18,95 \%$ \\
\hline \multirow{2}{*}{$\begin{array}{l}\text { Composición B } \\
\text { mezcla II }\end{array}$} & No.8 chino & $46,68 \mathrm{~mm}$ & $7,91 \%$ \\
\hline & $\begin{array}{l}\text { Sísmico } \\
\text { fuerza } 12\end{array}$ & $49,42 \mathrm{~mm}$ & $2,33 \%$ \\
\hline \multirow{2}{*}{$\begin{array}{l}\text { Composición B } \\
\text { mezcla III }\end{array}$} & $\begin{array}{l}\text { No. } 8 \\
\text { Chino } \\
\end{array}$ & $44,99 \mathrm{~mm}$ & $11,16 \%$ \\
\hline & $\begin{array}{l}\text { Sísmico } \\
\text { fuerza } 12\end{array}$ & $45,73 \mathrm{~mm}$ & $10,00 \%$ \\
\hline \multirow[b]{2}{*}{ Pentolita } & $\begin{array}{l}\text { No. } 8 \\
\text { Chino } \\
\end{array}$ & $38,59 \mathrm{~mm}$ & $22,15 \%$ \\
\hline & $\begin{array}{l}\text { Sísmico } \\
\text { fuerza } 12\end{array}$ & $36,98 \mathrm{~mm}$ & $25,89 \%$ \\
\hline \multirow{2}{*}{ PTX 2} & $\begin{array}{l}\text { No. } 8 \\
\text { Chino } \\
\end{array}$ & $43,82 \mathrm{~mm}$ & $12,21 \%$ \\
\hline & $\begin{array}{c}\text { Sísmico } \\
\text { fuerza } 12\end{array}$ & $43,53 \mathrm{~mm}$ & $12,30 \%$ \\
\hline
\end{tabular}

(*) El valor de \% de deformación es producto de réplicas de tres ensayos.

Para la cápsula sísmica fuerza 12 el de menor porcentaje de deformación fue composición B mezcla II con 2,33 \% y el de mayor deformación Pentolita con 25,89\%. Frente a las mezclas de composición B en ambos sistemas de iniciación chino y sísmico fuerza 12 presentaron mayor deformación del cilindro la mezcla I y la menor deformación la mezcla II.

Tabla 4. Resultados VoD

\begin{tabular}{|c|c|c|c|}
\hline EXPLOSIVO & $\begin{array}{l}\text { Tipo de } \\
\text { cápsula de } \\
\text { iniciación }\end{array}$ & $\begin{array}{c}\text { PESO } \\
\text { MUESTRA } \\
(\mathrm{g})\end{array}$ & $\begin{array}{l}\text { Velocidad de } \\
\text { detonación } \\
(\mathrm{m} / \mathrm{s})\end{array}$ \\
\hline \multirow{2}{*}{$\begin{array}{l}\text { Composición } \\
\text { B Mezcla I }\end{array}$} & $\begin{array}{c}\text { No. } 8 \\
\text { Fabricación } \\
\text { China } \\
\end{array}$ & $150 \mathrm{~g}$ & No registra \\
\hline & $\begin{array}{c}\text { Sísmico } \\
\text { fuerza } 12\end{array}$ & $150 \mathrm{~g}$ & 8835 \\
\hline \multirow[t]{2}{*}{ PTX-2 } & $\begin{array}{c}\text { No. } 8 \\
\text { Fabricación } \\
\text { China } \\
\end{array}$ & $150 \mathrm{~g}$ & 8820 \\
\hline & $\begin{array}{c}\text { Sísmico } \\
\text { fuerza } 12 \\
\end{array}$ & $150 \mathrm{~g}$ & 8265 \\
\hline \multirow{2}{*}{ PENTOLITA } & $\begin{array}{l}\text { No. } 8 \\
\text { Fabricación } \\
\text { China }\end{array}$ & $150 \mathrm{~g}$ & 7750 \\
\hline & $\begin{array}{c}\text { Sísmico } \\
\text { fuerza } 12\end{array}$ & $150 \mathrm{~g}$ & 8547 \\
\hline
\end{tabular}

(*) El valor de velocidad de detonación (VoD) producto de réplicas de tres ensayos.

La Tab.4 muestran las velocidades de detonación, tomando para esta prueba 150 gramos de explosivo, únicamente con los sistemas de iniciación chino y sísmico fuerza 12; presentando en general valores similares de VoD, para PTX2 y pentolita con estos dos sistemas de iniciación a excepción 
de composición B mezcla 1 por temas de no registro con la cápsula de iniciación china No. 8.

Tabla 5. Resultados prueba de péndulo

\begin{tabular}{|c|c|c|c|}
\hline Explosivo & $\begin{array}{l}\text { Tipo de } \\
\text { cápsula de } \\
\text { iniciación }\end{array}$ & $\begin{array}{l}\text { Longitud de } \\
\text { elongación } \\
(\mathrm{mm})^{*}\end{array}$ & $\begin{array}{l}\% \text { avance } \\
\text { respecto a } \\
\text { la Pentolita } \\
*\end{array}$ \\
\hline $\begin{array}{l}\text { Capsula de } \\
\text { Iniciación }\end{array}$ & $\begin{array}{l}\text { No. } 8 \\
\text { peruana }\end{array}$ & $8,38 \mathrm{~mm}$ & - \\
\hline $\begin{array}{l}\text { Composición } \\
\text { B mezcla I }\end{array}$ & $\begin{array}{l}\text { No. } 8 \\
\text { peruana }\end{array}$ & $52,86 \mathrm{~mm}$ & $76.68 \%$ \\
\hline $\begin{array}{l}\text { Composición } \\
\text { B mezcla II }\end{array}$ & $\begin{array}{l}\text { No.8 } \\
\text { peruana }\end{array}$ & $48,34 \mathrm{~mm}$ & $70,12 \%$ \\
\hline $\begin{array}{l}\text { Composición } \\
\text { B mezcla III }\end{array}$ & $\begin{array}{l}\text { No.8 } \\
\text { peruana }\end{array}$ & $31,32 \mathrm{~mm}$ & $45,43 \%$ \\
\hline PTX-2 & $\begin{array}{l}\text { No.8 } \\
\text { peruana }\end{array}$ & $62,54 \mathrm{~mm}$ & $90,71 \%$ \\
\hline Pentolita & $\begin{array}{l}\text { No.8 } \\
\text { peruana }\end{array}$ & $68,94 \mathrm{~mm}$ & $100 \%$ \\
\hline
\end{tabular}

Tabla 6. Resultados prueba de péndulo

\begin{tabular}{|c|c|c|c|}
\hline Explosivo & $\begin{array}{l}\text { Tipo de } \\
\text { capsula de } \\
\text { iniciación }\end{array}$ & $\begin{array}{l}\text { Longitud de } \\
\text { elongación }(\mathrm{mm})\end{array}$ & $\begin{array}{l}\% \text { avance } \\
\text { respecto a } \\
\text { la } \\
\text { Pentolita } \\
(*)\end{array}$ \\
\hline $\begin{array}{c}\text { Solo } \\
\text { detonador } \\
\end{array}$ & $\begin{array}{l}\text { No. } \\
\text { Chino }\end{array}$ & $8,38 \mathrm{~mm}$ & - \\
\hline \multirow{2}{*}{$\begin{array}{l}\text { Composición } \\
\text { B mezcla I }\end{array}$} & $\begin{array}{l}\text { No. } \\
\text { China }\end{array}$ & $47,36 \mathrm{~mm}$ & $103.05 \%$ \\
\hline & $\begin{array}{l}\text { Sísmico } \\
\text { fuerza } 12\end{array}$ & $45,00 \mathrm{~mm}$ & $97,91 \%$ \\
\hline \multirow{2}{*}{$\begin{array}{l}\text { Composición } \\
\text { B mezcla II }\end{array}$} & $\begin{array}{ll}\text { No. } & 8 \\
\text { China } & \end{array}$ & $43,28 \mathrm{~mm}$ & $94,16 \%$ \\
\hline & $\begin{array}{l}\text { Sísmico } \\
\text { fuerza } 12\end{array}$ & $40,72 \mathrm{~mm}$ & $88,60 \%$ \\
\hline \multirow{2}{*}{$\begin{array}{l}\text { Composición } \\
\text { B mezcla III }\end{array}$} & $\begin{array}{ll}\text { No. } & 8 \\
\text { China } & \\
\end{array}$ & $45,49 \mathrm{~mm}$ & $98,98 \%$ \\
\hline & $\begin{array}{l}\text { Sísmico } \\
\text { fuerza } 12 \\
\end{array}$ & $39,70 \mathrm{~mm}$ & $86,39 \%$ \\
\hline \multirow{2}{*}{ PTX-2 } & $\begin{array}{ll}\text { No. } & 8 \\
\text { China } & \end{array}$ & $46,57 \mathrm{~mm}$ & 101,33 \\
\hline & $\begin{array}{l}\text { Sísmico } \\
\text { fuerza } 12\end{array}$ & $48,16 \mathrm{~mm}$ & $104,80 \%$ \\
\hline \multirow{2}{*}{ Pentolita } & $\begin{array}{ll}\text { No. } & 8 \\
\text { China } & \\
\end{array}$ & $45,96 \mathrm{~mm}$ & $100 \%$ \\
\hline & $\begin{array}{l}\text { Sísmico } \\
\text { fuerza } 12\end{array}$ & $52,95 \mathrm{~mm}$ & $115,22 \%$ \\
\hline
\end{tabular}

* La longitud de desplazamiento y porcentaje de avance es estimada a partir del promedio de dos ensayos.

En la Tab. 5, se muestra los resultados de elongaciones de los materiales explosivos, tomando 10 gramos para la prueba de péndulo bajo la cápsula de iniciación No. 8 de fabricación peruana. El material explosivo más sensible a esta prueba es pentolita cuya longitud de elongación fue de $68,94 \mathrm{~mm}$ y el menos sensible son las mezclas de composición B, entre estas la mezcla III es la que presenta menor desplazamiento con longitud de elongación de 31,32 mm.
De otra parte, los datos de la Tab. 6, sensibilidad a otros sistemas de iniciación como el chino y el sísmico fuerza 12, muestra que los explosivos militares menos sensible y más sensible para la cápsula de iniciación no 8 de fabricación china son la composición B mezcla III con longitud de 45,49 mm y el más sensible, composición B mezcla I con longitud de $47,36 \mathrm{~mm}$.

Referente al sistema de iniciación fuerza 12 el más sensible de los explosivos es PTX-2 con longitud de 48,16 mm y el menos sensible es la composición B de la mezcla III con longitud de 39,70 mm. Sin embargo, al estimar los resultados de sensibilidad de estos materiales explosivos en los dos sistemas de iniciación peruano y sísmico fuerza 12, su longitud y rango de elongación en el péndulo comprenden un intervalo de $39,70 \mathrm{~mm}$ a $52,95 \mathrm{~mm}$. El anterior intervalo es más bajo si se compara el rango comprendido para estos materiales explosivos con relación al rango obtenido en la cápsula de iniciación peruana, cuyo intervalo de longitud es de $31,32 \mathrm{~mm}$ a $68,94 \mathrm{~mm}$.

\section{Discusión de resultados}

Efectivamente, los sistemas de iniciación o cápsulas influyen en la entrega de energía a un material explosivo afectando ellos el nivel de trituración de la demolición a realizar. Esto es evidenciado en los resultados obtenidos de las características técnicas, como fue la prueba de Hess, prueba de péndulo y velocidad de detonación.

La pentolita, por ejemplo, tiene mayor poder rompedor al emplear sistemas de iniciación de fuerza 12 y fabricación china en relación al de fabricación peruana. Estos datos guardan una correlación con la prueba de péndulo y velocidad de detonación, obteniéndose valores mayores de sensibilidad y velocidad de detonación al emplear el sistema de iniciación de fuerza 12.

El explosivo PTX-2; obtuvo buen poder rompedor con el sistema de iniciación peruano, no obstante, su sensibilidad (prueba péndulo) a este sistema de iniciación fue levemente menor en relación a los sistema de iniciación chino y fuerza 12. En relación a la velocidad de detonación, sus valores están en los rangos esperados, no obteniéndose entre sus sistemas de iniciación diferencias drásticas. Por consiguiente este explosivo en actividades de demolición debe ser usado con el sistema de iniciación peruano.

Las mezclas de composición $\mathrm{B}$, frente al ensayo de poder rompedor, la que mayor respuesta obtuvo a los sistemas de iniciación en este ensayo fue la mezcla 1 conformada esta por $69 \%$ de TNT, $39 \%$ RDX y $1 \%$ de cera. Las demás composiciones de mezclas de composición $\mathrm{B}$ sus resultados no fueron promisorios. Ahora bien, la mezcla-1 de composición B su mayor poder rompedor fue conseguido con el sistema de iniciación fuerza 12, posteriormente el chino y finalmente el peruano. En los ensayos de péndulo, su sensibilidad fue cercana en valores, mayor para el sistema de iniciación chino, levemente el de fuerza 12 y finalmente 
menor el peruano. En relación a la velocidad de detonación solo presento registro con el sistema de iniciación fuerza 12, y no con el sistema de iniciación chino; esto es debido probablemente a la mayor cantidad de explosivo a iniciar en este ensayo, para formar el régimen de la onda de choque.

En una demolición, a la hora de seleccionar un explosivo tipo composición $\mathrm{B}$, se debe seleccionar la mezcla 1 de composición B y como sistema de iniciación el de fuerza 12. Este hecho amerita el saber elegir componentes o sustancias químicas a la hora de formular explosivos a partir de otras sustancias químicas o explosivas.

\section{Conclusiones}

Los resultados experimentales obtenidos en la prueba de péndulo en sensibilidad para los explosivos militares favorecieron su sensibilidad a la cápsula de iniciación No. 8 de fabricación peruana en relación a las cápsulas de iniciación china no 8 y sísmica fuerza 12. La prueba de Hess determino la energía rompedora de un explosivo mediante la evaluación experimental de la presión de detonación, siendo esta decisiva para iniciar y evaluar las mezclas de composición B con los sistemas de iniciación chino y sísmico fuerza 12 y negativamente con la cápsula de iniciación No 8 de fabricación peruana. Los parámetros de sensibilidad y presión de detonación son complementarios y determina el éxito de detonación de un explosivo militar con mayor aprovechamiento de energía del mismo o para realizar un trabajo, viéndose estos resultados con éxito la elección de cápsulas de iniciación china y sísmica fuerza 12 para las mezclas de composición $\mathrm{B}$ y no con cápsula iniciación de fabricación peruana No 8.

Se determinó la velocidad de detonación (VoD) de los explosivos militares; siendo estos valores similares en VoD para PTX-2 y pentolita; confirmando la similitud de los resultados VoD con los obtenidos en el rango de deformación del cilindro para estos explosivos en la prueba de Hess.

\section{Referencias}

[1] Manual práctico de voladura EXSA, 2013.

[2] FM 5-250 Cuartel General, Secretaria del Ejército, 1998.

[3] Industria Militar Colombiana, 2016. Productos Fabrica explosivos Antonio Ricaurte.

[4] Instituto Tecnológico Geominero de España, 1994.

[5] R. Meyer- J. Kohler- A. Homburg. Explosives. 6th edición.2007.

[6] Basil T. Fedoroff. Henry A. Aaronson. Earl F. Reese. Oliver E. Sheffield. George D. Clift. Encyclopedia of explosives and related items U.S. Army research and development command Tacom, Ardec. New Jersey, USA 1960

[7] Urbanski Tadeusz. Chemistry and technology of explosives. PWNPolish Scientific Publishers Warszawa 1964.

[8] J Rincon, J Fonseca; "Calculo de parámetros termodinámicos para explosivos militares. Aplicación de fundamentos termodinámicos y propiedades de los explosivos militares". Rev.Cient.Gen. José María Córdova, Vol 13, pp 221-239, Jul-Dic.2015

[9] Manual de empleo de explosivos. Unión española de explosivos. Imprime graficas Rogar s.a Navalcarnero, Madrid 2002.
[10] Military explosives. Department of the Army Technical. Manual TM 91300-214. Headquarters, Department of the Army, September 1984.

[11] W. Cooper Paul. Explosives Enngineering Library of Congress Cataloging in Publication Data. Wiley-VCH 1996. 